\title{
Filles, garçons : quel capital émotionnel pour quelles conséquences?
}

\section{Bénédicte Gendron}

\section{(2) OpenEdition \\ 1 Journals}

\section{Édition électronique}

URL : http://journals.openedition.org/trema/1095

DOI : 10.4000/trema.1095

ISSN : 2107-0997

\section{Éditeur}

Faculté d'Éducation de l'université de Montpellier

\section{Édition imprimée}

Date de publication : 1 juin 2010

Pagination : $39-47$

ISSN : 1167-315X

\section{Référence électronique}

Bénédicte Gendron, «Filles, garçons : quel capital émotionnel pour quelles conséquences ? », Tréma

[En ligne], 32 | 2010, mis en ligne le 01 juin 2012, consulté le 30 avril 2019. URL : http:// journals.openedition.org/trema/1095; DOI : 10.4000/trema.1095

Ce document a été généré automatiquement le 30 avril 2019.

Trema 
Filles, garçons : quel capital émotionnel pour quelles conséquences?

\author{
Bénédicte Gendron
}

I. Introduction 
1 Jules FERRY dans un discours à l'Assemblée nationale avançait déjà le 10 avril 1870 : « Avec l'inégalité d'éducation, je vous défie d'avoir jamais l'égalité des droits, non l'égalité théorique, mais l'égalité réelle.». Si l'éducation est un droit, elle n'est, en effet, pas égale et identique pour tous. Déjà, l'éducation est un lieu propice à la reproduction des normes liées aux rôles sociaux de sexe. Est-ce l'origine des inégalités en œuvre entre hommes et femmes? L'éducation est-elle la même pour tous? Éduque-t-on de la même façon ou développe-t-on les mêmes compétences chez les filles et les garçons? Si nombre de travaux ont mis en évidence des différences de dotation en capital humain entre les filles et les garçons, renvoyant essentiellement aux savoirs et savoir faire, encore peu de travaux se sont intéressés aux savoir - être et aux compétences émotionnelles. La séparation

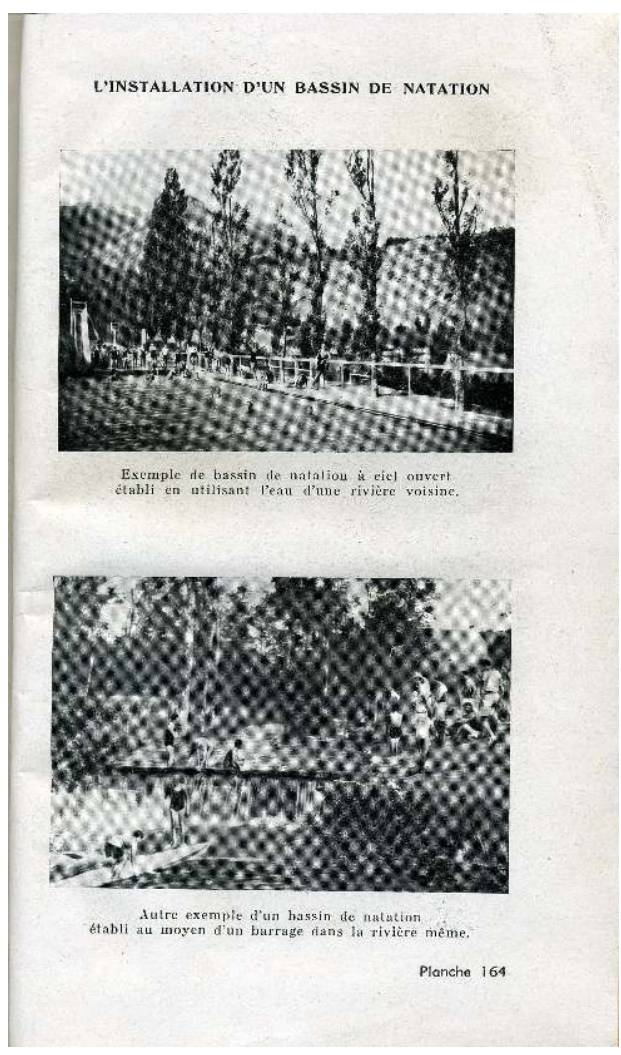
traditionnelle des domaines cognitif et conatif a longtemps empêché de prendre toute la mesure de la place que le savoir - être occupe dans la formation des personnes et de ses incidences sur leurs devenirs. Cet article se propose d'étudier l'impact de l'éducation sexuée ${ }^{1}$ sur le développement des compétences émotionnelles des filles et des garçons, participant à la constitution d'un capital émotionnel différencié. Après avoir défini la notion de capital émotionnel et ses caractéristiques et rendu compte du profil du capital émotionnel des filles et des garçons, on analysera les différences de performances scolaires et des choix d'orientation scolaire et professionnelle à travers le prisme des compétences émotionnelles.

\section{Des compétences émotionnelles au capital émotionnel}

2 Les recherches récentes en psychologie, en neuropsychologie apportent de nouveaux éclairages sur les émotions et les compétences associées à leurs régulations. Ces avancées suscitent de nouvelles réflexions et de nouveaux ponts entre disciplines (psychologie de l'éducation et économie de l'éducation) dont le capital émotionnel se veut une tentative et l'objet de cet essai conceptuel.

\section{1. Régulation et compétences émotionnelles}

\section{1. 1. Emotion et régulation émotionnelle}

3 Selon DAMASIO (1994), loin de constituer un obstacle à la prise de décision rationnelle dans la vie quotidienne, les émotions en permettant l'harmonisation des différents 
processus cognitifs se révèleraient être la condition indispensable d'adaptation et de réaction optimale à une situation donnée. Décisives pour l'adaptation de l'individu et ceci, dès sa naissance jusqu'à l'âge adulte à des degrés divers, elles continuent à l'être, par la suite, en tant qu'adaptations circonstanciées à des modèles sociaux. Aussi, cela justifie que les personnes aient besoin d'apprendre des autres pour savoir comment gérer leur environnement et eux-mêmes. C'est ici qu'interviennent la régulation émotionnelle et particulièrement l'intelligence et les compétences émotionnelles qui lui sont rattachées.

\section{1. 2. Intelligence émotionnelle (IE) et compétences émotionnelles}

4 Parmi les modèles d'IE, le modèle de GOLEMAN et CHERNISS (2001) qui reprend les apports des travaux de SALOVEY et MAYER, définit l'IE comme la capacité à reconnaître et à maîtriser les émotions en soi et chez les autres.

5 Elle est décomposée en quatre principaux concepts déclinés en compétences: la conscience de soi ou autoévaluation (capacité à comprendre ses émotions et à reconnaître leur incidence), la maîtrise de soi ou autorégulation (capacité à maîtriser ses émotions et impulsions et à s'adapter à l'évolution de la situation), la conscience sociale ou empathie (capacité à détecter et à comprendre les émotions d'autrui et à y réagir tout en comprenant les réseaux sociaux) et la gestion des relations ou aptitudes sociales de communication (qui correspond à la capacité à inspirer et à influencer les autres tout en favorisant leur développement et en gérant les conflits). Au lieu de les appréhender comme dans certaines recherches en termes de "qualités ", « dispositions individuelles » ou encore "d'attributs", "traits » interpersonnels et intra personnels, le modèle de GOLEMAN les analyse en terme de compétences permettant des rapprochements avec l'économie des ressources humaines où le concept de compétences renvoie non pas à des aptitudes génétiques, « innées » ou encore « naturelles » mais résultant d'expériences et d'apprentissages.

\section{2. Essai de définition du capital émotionnel et de ses caractéristiques}

\section{2. 1. Définition}

6 Dans tous les champs de l'activité humaine, entre autres dans l'éducation, à l'école ou encore au travail où les émotions sont présentes (secteurs de la santé, de l'éducation, du social ...), les compétences émotionnelles sont utiles et même cruciales. Elles constituent une ressource pour les personnes dans la constitution et l'utilisation optimale du capital humain. Issues d'un apprentissage précoce et se développant tout au long des expériences de vie, ces compétences émotionnelles varient d'une personne à l'autre, certains étant mieux dotés que d'autres ou ayant développé un capital plus équilibré que d'autres. Elles constituent un réel capital qu'il importe de développer, d'entretenir du fait que le capital émotionnel est l'ensemble des ressources - renvoyant aux compétences émotionnelles inhérentes à la personne, utiles au développement personnel, professionnel et organisationnel (Gendron, 2008) participant à la constitution et l'utilisation optimale du capital humain, du capital social et entre autres, à la cohésion sociale. 


\section{2. 2. Caractéristiques}

7 Le capital émotionnel et ses compétences associées interviennent dans le procès de constitution du capital humain. Il constitue les pré - requis du développement cognitif. IZARD, KAGAN et ZAJONC (1984), du point de vue de l'apprentissage, considèrent la conation et la cognition dans un rapport «synergiste ». Ces compétences s'avèreraient nécessaires et cruciales pour autoriser l'apprentissage en situation de classe et des interactions adéquates. Dans l'interaction pédagogique, elles facilitent ou handicapent l'apprentissage et donc, à terme la constitution du capital humain. Aussi, ce capital dépend des contextes et du milieu social des expériences de vie de la personne.

Un des contextes importants a longtemps placé la famille comme premier cadre de socialisation et de référence de construction identitaire de l'enfant. Cependant, celle-ci connaît aujourd'hui des bouleversements (divorce, famille monoparentale, recomposée ... ) où les normes éducatives et l'espace de référence se voient modifiés et diversifiés. La diversité des cadres premiers de socialisation conduit aujourd'hui à une hétérogénéité (différence de valeurs, de normes ...) et une diversité importante parmi les enfants en terme de dotation en capital émotionnel. Et ces différences en dotation peuvent venir freiner, altérer l'apprentissage, voire ne pas l'autoriser et influer sur le procès de constitution du capital humain. En effet, le capital émotionnel est plus qu'une variable additionnelle. Il est un «booster » dans le sens où il permet de potentialiser le capital humain et social. Il est essentiel pour utiliser de manière optimale et maximale les deux autres capitaux. Du point de vue de sa relation au capital humain, nombre de travaux mettent en évidence les préjudices d'une mauvaise gestion de ses émotions sur le développement, sur l'apprentissage, sur l'action ou encore sur la prise de décision (LAZARUS et FOLKMAN, 1984). A l'inverse, pour COSTALAT (1997), des émotions bien gérées permettent d'atteindre un optimum émotionnel autorisant une utilisation optimale de ses ressources et capacités. Également, une faible autoévaluation de soi ou estime de soi peut amener la personne à ne pas utiliser de manière maximale son potentiel et capital humain. C'est ce que soulignent les travaux en psychologie sur les attributions causales ${ }^{2}$, de locus of control ou en particulier, ceux sur le sentiment d'efficacité personnelle (voir BANDURA, 1997, pour une revue). Ils montrent que les personnes s'investissent rarement dans une activité qu'elles ne s'estiment pas en mesure de réaliser ou dans lesquelles elles se sentent peu efficaces. En cela, la compétence d'autoévaluation est cruciale. C'est ici qu'elle joue pleinement son rôle, soit de booster si l'estime et la confiance en soi sont fortes, soit de réducteur ou d'inhibiteur si l'évaluation de soi et de ses capacités dans un domaine donné est défavorable ou sous-estimée. Dès lors, une évaluation correcte ou faussée de soi ${ }^{3}$ conditionnera la mobilisation maximale ou non de son potentiel en capital humain. En outre, le capital émotionnel importe également dans la construction du capital social. Un capital émotionnel social développé participe de l'intégration sociale et de la constitution du capital social. La constitution et mobilisation du capital social que la personne pourra mobiliser en temps et à des fins utiles va dépendre de ses compétences émotionnelles sociales, précisément de ces aptitudes sociales de communication et de sa conscience sociale. Si les liens sociaux participent à la production du capital social, leur mobilisation ou utilisation comme «capital » dépend de sa conscience de soi, particulièrement son évaluation de soi : sa confiance et son estime de soi. 


\section{Capital émotionnel et essai d'analyse des différences de performances scolaires et d'orientation scolaires et professionnelles entre les filles et les garçons}

9 La production du capital émotionnel étant le fait, entre autres, de l'éducation et d'expériences de vie, quel est l'impact de dotations différentes au niveau individuel ? De plus, ce capital est-il identique entre filles et garçons? Peut-il être un élément d'explication des différences observées entre filles et garçons en termes de réussite scolaire et d'orientation scolaire et professionnelle? Après avoir rendu compte de la méthodologie, l'approche par le capital émotionnel entend apporter quelques éléments de réponses à ces questions.

\section{1. Méthodologie et données}

10 S'inspirant d'une approche méta analytique de revue de la littérature scientifique nationale et internationale sur le genre, il a été dressé un tableau des compétences émotionnelles développées chez les filles et les garçons. Précisément, les profils caractéristiques alloués dans la littérature scientifique aux filles et aux garçons énoncés en termes d'attributs, de traits de personnalité, d'attitudes, ou de comportements... ont été appréhendés comme des compétences émotionnelles.

Tableau 1 Capital émotionnel : Différences Filles - Garçons

\begin{tabular}{|c|c|c|c|c|c|}
\hline $\begin{array}{c}\text { Compétences émotionnelles } \\
\text { personnelles }\end{array}$ & Garçons & Filles & $\begin{array}{c}\text { Compétences } \\
\text { émotionnelles sociales }\end{array}$ & Garçons & Filles \\
\hline Auto-évalutation & + haute & $\begin{array}{c}+ \\
\text { basse }\end{array}$ & Empathie & + basse & $\begin{array}{c}+ \\
\text { haute }\end{array}$ \\
\hline Auto-régulation & + basse & $\begin{array}{c}+ \\
\text { haute }\end{array}$ & $\begin{array}{c}\text { Aptitudes sociales de } \\
\text { communication }\end{array}$ & + basse & + \\
haute \\
\hline
\end{tabular}

11 Partant de cette grille d'analyse par les compétences, les profils en compétences émotionnelles des filles et des garçons ont été définis relativement à l'un des deux sexes : plus fortement (+haute) ou plus faiblement (+basse) développées chez le garçon/la fille comparativement à la fille/au garçon (tableau 1). 


\title{
III. 2. Essai d'analyse des différences filles-garçons en éducation et dans l'orientation scolaire et professionnelles à partir du capital émotionnel
}

\author{
III. 2. 1. Le capital émotionnel essentiel à la constitution du capital humain de \\ départ : l'avantage des filles à l'école (traditionnelle) et leur meilleure performance \\ scolaire
}

12 L'école traditionnelle requiert un certain nombre de dispositions et comportements « scolaires » renvoyant à certaines compétences émotionnelles essentielles pour réussir scolairement dans de tels systèmes. Aussi, par l'éducation différenciée reçue depuis leur plus jeune âge, les garçons et les filles en sont dotés différemment. En effet, si l'environnement social joue un rôle important dans l'éducation, déjà l'environnement familial, la famille, les parents n'éduquent pas les filles et les garçons de la même façon (MOSCONI, 1994) et participent en cela à la différenciation du capital émotionnel. Ainsi, les parents ont en effet tendance à plus encourager leurs enfants mâles à défendre leurs intérêts et à affirmer leur personnalité qu'ils ne le font pour leurs filles. Les garçons seraient "habitués " dès le plus jeune âge à prendre plus de risques physiques (comportements " casse-cou ») et sociaux (goût de l'affrontement et de la compétition) que les filles. À l'inverse, les filles se voient encouragées à être dociles, obéissantes; et leur timidité est mieux supportée par les parents. Elles se révèlent davantage " préoccupées » par les échanges, le respect et la diffusion des règles sociales. Ainsi, elles seraient plus conformistes ou « socialement formées à » se conformer, plus respectueuses des règles, seraient moins assertives que les garçons (mise en avant moins forte, plus basse estime de soi...) (ANDRE et LELORD, 2002, et LORANGER, 1988). Autant de comportements peu propices au développement d'une estime de soi forte et stable mais cependant, favorables au développement des compétences d'écoute, et d'autorégulation (CURNOYER, SOLOMON and TRUDEL, 1998; ROTHBART, 1989); en cela, elles répondent mieux que les garçons aux comportements attendus à l'école traditionnelle. Ainsi, certaines compétences émotionnelles développées chez les filles leur procureraient un avantage relativement aux standards et programmes scolaires et correspondraient plus largement aux dispositions scolaires attendues et requises pour "réussir " à l'école traditionnelle (FELOUZIS, 1993). Au-delà d'autres variables traditionnellement avancées, le capital émotionnel pourrait ainsi venir apporter des éléments d'explication à la meilleure performance scolaire des filles relativement à celle des garçons à l'école traditionnelle.

\section{2. 2. Le capital émotionnel et l'orientation scolaire et professionnelle : l'avantage des garçons dans la compétition}

13 Les garçons et les filles n'entrent pas dans les situations d'apprentissage de manière identique, ne vivent pas les mêmes expériences et se construisent différemment de par l'éducation sexuée dès leur plus jeune âge. Et, dès lors, ils ne sont pas préparés de manière identique aux différentes orientations scolaires et professionnelles, particulièrement pour les filières compétitives. Les enseignants participent aussi à cette différenciation, particulièrement au développement de ces compétences personnelles chez les garçons. En effet, la plus grande agressivité chez les garçons, leur plus faible 
conformisme et leur comportement énergique vont induire de manière involontaire et inconsciente des comportements d'enseignants non équitables en leur faveur cette fois (MOSCONI, 1997 ; DURU-BELLAT, 1994 et 1998) ; par exemple, une plus fréquente prise de parole donnée aux garçons participe au développement et renforcement des compétences émotionnelles personnelles des garçons et corollairement, peut contribuer au non développement ou à l'atrophie de ces compétences chez les filles. Or ces compétences émotionnelles personnelles s'avèrent très utiles pour accéder aux filières scolaires compétitives et plus tard, pour accéder à l'emploi et aux postes à responsabilité sur le marché du travail. Ainsi, de par leur capital émotionnel personnel (assertivité, confiance en soi, sentiment d'efficacité...) plus développé, les garçons seraient mieux préparés que les filles à faire face à la compétition dans les filières dites "prestigieuses ou de compétition ». Les recherches sur l'auto - efficacité personnelle montrent que les garçons ont une meilleure estime d'eux-mêmes relativement aux filles et une meilleure capacité à s'auto - évaluer et une tendance à se surévaluer lorsqu'il s'agit d'estimer leur chance de réussite dans les filières scolaires compétitives. Cette compétence émotionnelle «personnelle » forte est à leur avantage car elle limite l'auto - censure et motive les garçons à candidater dans les filières de compétition ou des carrières ambitieuses plus fréquemment que les filles (BEYER, 1991, BAUDELOT et ESTABLET, 1992, GENDRON et LAFORTUNE, 2009).

Chez les filles, le capital émotionnel développé (empathie, souci du service, de l'écoute ...) participe à leur orientation vers des filières à caractère "social» ou vers des filières d'» humanités »; à l'inverse, leur faible dotation en capital émotionnel "personnel » pourrait venir expliquer la faiblesse numérique des filles dans les filières compétitives que sont les filières scientifiques ${ }^{4}$. En effet, les filles étant moins éduquées et socialisées à être dans la compétition (cf. Le curriculum caché ou - l'éducation implicite - POURTOIS et DESMET, 2004), elles tendent à s'autocensurer en ne se présentant pas dans les filières prestigieuses scientifiques ou en ne s'engageant pas dans des filières ambitieuses ou de compétition, ou encore en hésitant à se présenter dans des filières professionnelles trop connotées «masculines » ou non - féminines. D'ailleurs, certaines filières scolaires ellesmêmes peuvent participer à la sexuation des orientations. En outre, des mécanismes de différenciation de genre jouent fortement et particulièrement en période d'adolescence qui correspond à la période d'orientation scolaire et professionnelle. En effet, dans cette période se construit et s'affirme, par le biais des représentations, l'identité sexuée. Et «ces représentations que les adolescents ont des rôles adultes dépendent évidemment des modèles que le milieu leur propose et des valeurs qu'il leur transmet » (ZAZZO, 1966, p.20). Ainsi, des modèles de division sexuée en action dans la sphère sociale vont s'imposer à elles comme forme légitime de division. Transmis dès le plus jeune âge et se continuant via l'éducation et la socialisation, ceux-ci prescrivent des codes sociaux de comportements répondant aux normes culturelles et sociales de répartition des rôles sociaux de sexe (CHODOROW, 1988, GILLIGAN, 1982, Le MANER-IDRISSI and al. 2002, ZAOUCHE-GAUDRON et ROUYER, 2002).

\section{Conclusion}

Enfin, selon le capital émotionnel développé, précisément s'il relève d'une éducation sexuée, les filles et les garçons ne sont pas "également " préparés pour les différentes orientations scolaire et professionnelle dans le système d'enseignement. Déjà bien ancrée 
avant l'entrée en classe secondaire, cette éducation différenciée vient conditionner les choix d'orientation. En cela, il importe, au-delà des campagnes de sensibilisation « des filles en filières scientifiques et techniques ", d'informer les parents et former le personnel éducatif (GENDRON et LAFORTUNE, 2009) à une éducation équitable où les compétences émotionnelles seraient prises en compte et autoriseraient par une éducation consciente des stéréotypes sociaux de sexe un «réel » choix des filles et les garçons de leur orientation scolaire et professionnelle.

\section{BIBLIOGRAPHIE}

ANDRE, C. et LELORD, F. (2002), L'estime de soi, s'aimer pour mieux vivre avec les autres, Paris : Odile JACOB.

BANDURA, A. (1977), Self-efficacy : toward a unifying theory of behavioral change, Psychological Review, 84 (2), 191-215.

BAUDELOT, C., \& ESTABLET, R. (1992), Allez les filles ! Paris : Seuil.

BAUDELOT, C. \& ESTABLET, R. (2007). Quoi de neuf chez les filles ? Entre stéréotypes et libertés. Paris : Nathan.

BEYER, S. (1991). The Effect of Self-consistency on Gender Differences in the Accuracy of selfevaluations. Dissertation, University of Oregon.

BRESSOUX, P. \& PANSU, P. (2003). Quand les enseignants jugent leurs élèves. Paris : Presses Universitaires de France.

CHILAND, C. (1999). Le sexe mène le monde. Paris : Calmann-Lévy.

CHODOROW, N. (1988). Femininum - maskulinum. Modersfunktion och könssociologi. Stockholm : Natur \& Kultur.

COSTALAT-FOUNEAU, A-M. (1997), Identité sociale et dynamique représentationnelle, Rennes : Presses Universitaires de Rennes.

CURNOYER, M. , SOLOMON, C. R. \& TRUDEL, M. (1998). I speak then I expect : Language and selfcontrol in the young child at home. Canadian Journal of Behavioral Science, 30, 69-81.

DAMASIO, A. (1994) Descartes'Error : Emotion, reason, and the human brain, New York : G.P. Putman's sons.

DURU-BELLAT, M. (1998), La mixité, un aspect du « curriculum caché » des élèves, Enfance et Psy, n³, 73-78.

DURU-BELLAT, M. (1994), Filles et garçons à l'école, approches sociologiques et psycho-sociales, Revue Française de Pédagogie, n¹09, octobre - novembre - décembre, 111-135.

DURU-BELLAT, M. (1990), L'école des filles, Quelles formations pour quels rôles sociaux ? Paris : L'Harmattan.

FELOUZIS, G. (1993), Interactions en classe et réussite scolaire, une analyse des differences filles garçons, Revue française de sociologie, 34, 2, p. 199-222.

GENDRON, B. \& LAFORTUNE, L. (dir.) (2009), Leadership et compétences émotionnelles dans l'accompagnement au changement, Ouvrage Collection Education \& Recherche, Québec : Presses de l'Université du Québec, p. 237. 
GENDRON , B. (2008), « Capital émotionnel et éducation », Dictionnaire de l'éducation, Van ZANTEN A. (dir.), Paris : PUF, p. 41-43.

GILLIGAN, C. (1982). In a Different Voice. Cambridge : Harvard University Press.

GOLEMAN, D. (2001), An EI-Based Theory of Performance, in The Emotionally Intelligent Workplace, C. Cherniss and D. Goleman Eds.

GOLEMAN, D. (1997), L'intelligence émotionnelle, Comment transformer ses émotions en intelligence, Paris : Robert Laffont.

LE MANER-IDRISSI, G., LEVEQUE, A., \& MASSA, J. (2002), Manifestations précoces de l'identité sexuée. L'orientation scolaire et professionnelle, $n^{\circ} 31$ (4), 507-522.

IZARD, C., KAGAN, J., \& ZAJONC, R. (1984). Emotions, cognition, and behavior. New York : Cambridge University Press.

LAZARUS R. S. et FOLKMAN S. (1984). Stress, appraisal and coping, New-York : Springer.

LORANGER, M. (1988), Les garçons et les filles en situation d'apprentissage, Druning, R.E Tremblay, Relations entre enfants : recherches et interventions éducatives, Paris : Fleurus.

MACCOBY, E.E. (Ed) (1966), The development of sex differences, Stanford, California : Stanford University Press.

MAYER, J.D. \& SALOVEY, P. (1993), The intelligence of emotional intelligence. Intelligence, 17, 433-442.

MAYER, J.D. \& SALOVEY, P. (1997), What is emotional intelligence?

P. SALOVEY \& D. SLUYTER (Eds). Emotional Development and Emotional Intelligence :

Implications for Educators, 3-31. New York : Basic Books.

MONTEIL, J. M. \& HUGUET, P. (2002), Réussir ou échouer à l'école : une question de contexte? Grenoble : Presses Universitaires de Grenoble.

MOSCONI, N. (2001), Comment les pratiques enseignantes fabriquent-elles de l'inégalité entre les sexes ? Séminaire du Cref, Equipe « Savoirs et rapport au savoir », mars. Paris X-Nanterre.

MOSCONI, N., (1999), « La mixité scolaire : enjeux sociaux et ethico-politiques », Télémaque, juin. MOSCONI, N. (1998), Égalité des sexes en éducation et formation, Paris : PUF.

MOSCONI, N. (1994), Femmes et savoir, La société, l'école et la division sexuelle des savoirs, Paris : L'Harmattan.

MOSCONI, N. (1989), La mixité dans l'enseignement secondaire : un faux-semblant ? Paris : PUF.

MOSCONI, N. \& LOUDET-VERDIER, J. (1997), Inégalités de traitement entre les filles et les garcons, in BLANCHARD-LAVILLE (sous la dir.), Variations sur une leçon de mathématiques. Analyses d'une séquence : l'écriture des grands nombres, Paris : L'Harmattan.

POURTOIS, J-P. \& DESMET, H. (2004), L'éducation implicite, Paris : PUF.

ROTHBART, M. K. (1989). Temperament and development. In G. A. Kohnstramm. J. A. BATES, and ROTHBART , M. K. (Eds.), Temperament in childhood, New York : Wiley, 59-73.

VOUILLOT, F., BLANCHARD, S., MARRO, C., \& STEINBTUCKNER M-L., (2004), La division sexuée de l'orientation et du travail : une question théorique et une question de pratiques, L'Orientation scolaire et professionnelle, $\mathrm{n}^{\circ} 10, \mathrm{pp} .277-291$. 
ZAOUCHE-GAUDRON, C., \& ROUYER, V. (2002), L'identité sexuée du jeune enfant : actualisation des modèles théoriques et analyse de la contribution paternelle. L'Orientation Scolaire et Professionnelle, $\mathrm{n}^{\circ} 31$ (4), 523-533.

ZAZZO, B. (1966), Psychologie différentielle de l'adolescence, Paris : PUF.

\section{NOTES}

1. Renvoi à l'identité sexuée définie par Christian BAUDELOT et Roger ESTABLET et reprenant celle de Colette CHILAND : l'identité de genre ou l'identité sexuée est «le sentiment intime que chacun a d'appartenir à l'un des sexes que la biologie et la culture distinguent » (CHILAND, 1999 ; BAUDELOT \& ESTABLET, 2007).

2. Façon dont les individus attribuent des causes aux événements et aux comportements, et assignent des causes et des responsabilités à différents aléas.

3. On retrouve cette idée à travers les concepts de "clairvoyance cognitive " et d' "acuité représentationnelle » chez COSTALAT-FOUNEAU, (1997, p. 50-51). L'acuité représentationnelle a un impact sur la représentation de soi et par conséquent dans la dynamique des compétences subjectives et objectives de l'individu qui mobilisent l'individu dans un projet d'action.

4. Et quand elles accèdent à ces dernières, elles tendent à s'orienter vers les sections ou options où la dimension « humaine » ou « vivante » est présente ; comme en « biologie » ou plus tard par exemple en filière de médecine mais moins souvent en mathématique ou physique (d'ailleurs ne serait-ce pas l'illustration du problème de la déshumanisation de certains curricula en sciences «dures»?)

\section{RÉSUMÉS}

Si l'éducation est un droit, elle n'est, en effet, pas égale et identique pour tous. Déjà, l'éducation est un lieu propice à la reproduction des normes liées aux rôles sociaux de sexe. Si nombre de travaux ont mis en évidence des différences de dotation en capital humain entre les filles et les garçons, renvoyant essentiellement aux savoirs et savoir-faire, encore peu de travaux se sont intéressés aux savoir - être et aux compétences émotionnelles. La séparation traditionnelle des domaines cognitif, affectif, et psychomoteur a longtemps empêché de prendre toute la mesure de la place que le savoir - être occupe dans la formation des personnes et de ses incidences sur leurs devenirs. Dans cette article, l'impact de l'éducation sexuée sur le développement des compétences émotionnelles des filles et des garçons, participant à la constitution d'un capital émotionnel différencié est étudiée et tente d'apporter des éléments d'éclairage aux différences observées en termes de performances scolaires et des choix d'orientation scolaire et professionnelle.

If education is a right, it is indeed not equal and identical for all. Already, education is a place conducive to the reproduction of social norms connected to gender roles. While many studies have highlighted differences in human capital endowment between girls and boys, referring mainly to knowledge and skills, few studies have been interested in savoir - être (attitudes) and emotional skills. The traditional separation of the cognitive, emotional and psychomotor 
domains has long prevented the full measure of the role that savoir-être has in the formation of persons and its implications for their futures. In this article, the impact of gender education on the development of emotional skills of girls and boys, involved in the formation of differentiated emotional capital is studied in the attempt to bring to light the elements of differences observed in terms of school performance and choices in educational and professional orientation.

INDEX

Mots-clés : capital émotionnel, carrière scolaire, éducation sexuée, fille, garçon, genre, orientation, performance scolaire

Keywords : boy, counselling, emotional capital, gender, gender education, girl, school career, school performance

\section{AUTEUR}

\section{BÉNÉDICTE GENDRON}

Professeur des universités, Université Montpellier III, benedicte.gendron@univ-montp3.fr 